\title{
Formation of a strong southward IMF near the solar maximum of cycle 23
}

\author{
S. Watari ${ }^{1}$, M. Vandas ${ }^{2}$, and T. Watanabe ${ }^{3}$ \\ ${ }^{1}$ Communications Research Laboratory, 4-2-1 Nukuikita, Koganei, Tokyo 184-8795, Japan \\ ${ }^{2}$ Astronomical Institute, Bocni II 1401, 14131 Praha 4, Czech Republic \\ ${ }^{3}$ Ibaraki University, 2-1-1 Bunkyo, Mito, Ibaraki 310-8512, Japan
}

Received: 10 January 2003 - Revised: 7 June 2003 - Accepted: 23 June 2003 - Published: 1 January 2004

\begin{abstract}
We analyzed observations of the solar activities and the solar wind parameters associated with large geomagnetic storms near the maximum of solar cycle 23 . This analysis showed that strong southward interplanetary magnetic fields (IMFs), formed through interaction between an interplanetary disturbance, and background solar wind or between interplanetary disturbances are an important factor in the occurrence of intense geomagnetic storms. Based on our analysis, we seek to improve our understanding of the physical processes in which large negative $B_{z}$ 's are created which will lead to improving predictions of space weather.
\end{abstract}

Key words. Interplanetary physics (Flare and stream dynamics; Interplanetary magnetic fields; Interplanetary shocks)

\section{Introduction}

According to Tsurutani and Gonzalez (1997 and references therein), interplanetary events with interplanetary magnetic fields (IMFs), $B_{z}$, of less than $-10 \mathrm{nT}$ and a duration longer than three hours, tend to cause intense geomagnetic storms. Strong southward IMFs are an important factor in the occurrence of geomagnetic storms. Tsurutani et al. (1988) have made a study of the causes of the southward $B_{z}$ component responsible for major geomagnetic storms near the previous solar maximum (1978-1979). From their analysis of 10 cases they concluded that the negative $B_{z}$ was caused by internal fields of ejecta for only four cases. For others there were various causes such as sheath field ahead of ejecta or shock intensification of pre-existing IMF. McComas et al. (1989) studied $B_{z}$ perturbations induced by a draped magnetic field ahead of fast coronal mass ejections (CMEs). Wu, Dryer, and Wu (1996) and Wu et al. (1996) suggested the formation of $B_{z}$ caused by interplanetary shocks using threedimensional MHD simulation. On the other hand, Marubashi
(1997 and references therein) noted the importance of interplanetary magnetic flux ropes as a source of geomagnetic storms. Cane, Richardson, and St. Cyr (2000) studied all halo/partial, halo coronal mass ejections (CMEs) detected by the LASCO coronagraphs on board the SOHO spacecraft during 1996-1999 and compared them to geomagnetic storms. They noted that the southward IMF associated with ejecta determined mainly their geo-effectiveness, regardless of whether ejecta was a magnetic cloud structure or not.

We studied the interplanetary disturbances with strong southward IMF near the maximum of solar cycle 23. Almost continuous observations of solar wind and solar activities from space near the maximum of solar cycle 23 enabled us to study the relation between solar activities and strong southward IMF. Our analysis is complementary to the study made by Tsurutani et al. (1988) for the previous solar cycle. However, our study includes more cases.

\section{Data sources}

We analyzed geomagnetic storms with $D_{s t}$ indices of less than $-100 \mathrm{nT}$ between 1998 and 2000. There were 30 such cases which satisfied this criterion. In our analysis, hourly averaged solar wind parameters (magnetic field, speed, density, and temperature) observed by the ACE spacecraft were used to study the cause of strong southward IMFs associated with the geomagnetic storms. The solar observations of the SOHO and Yohkoh spacecrafts were used to identify the solar sources of the geomagnetic storms.

Table 1 lists the starting dates of magnetic storms, types of storms, minimum values of $D_{s t}$ index and minimum values of $B_{z}$ during the storms, the causes of strong southward IMFs, solar sources of the geomagnetic storms, occurrence dates of the solar sources, and transit speeds of the interplanetary disturbances. 


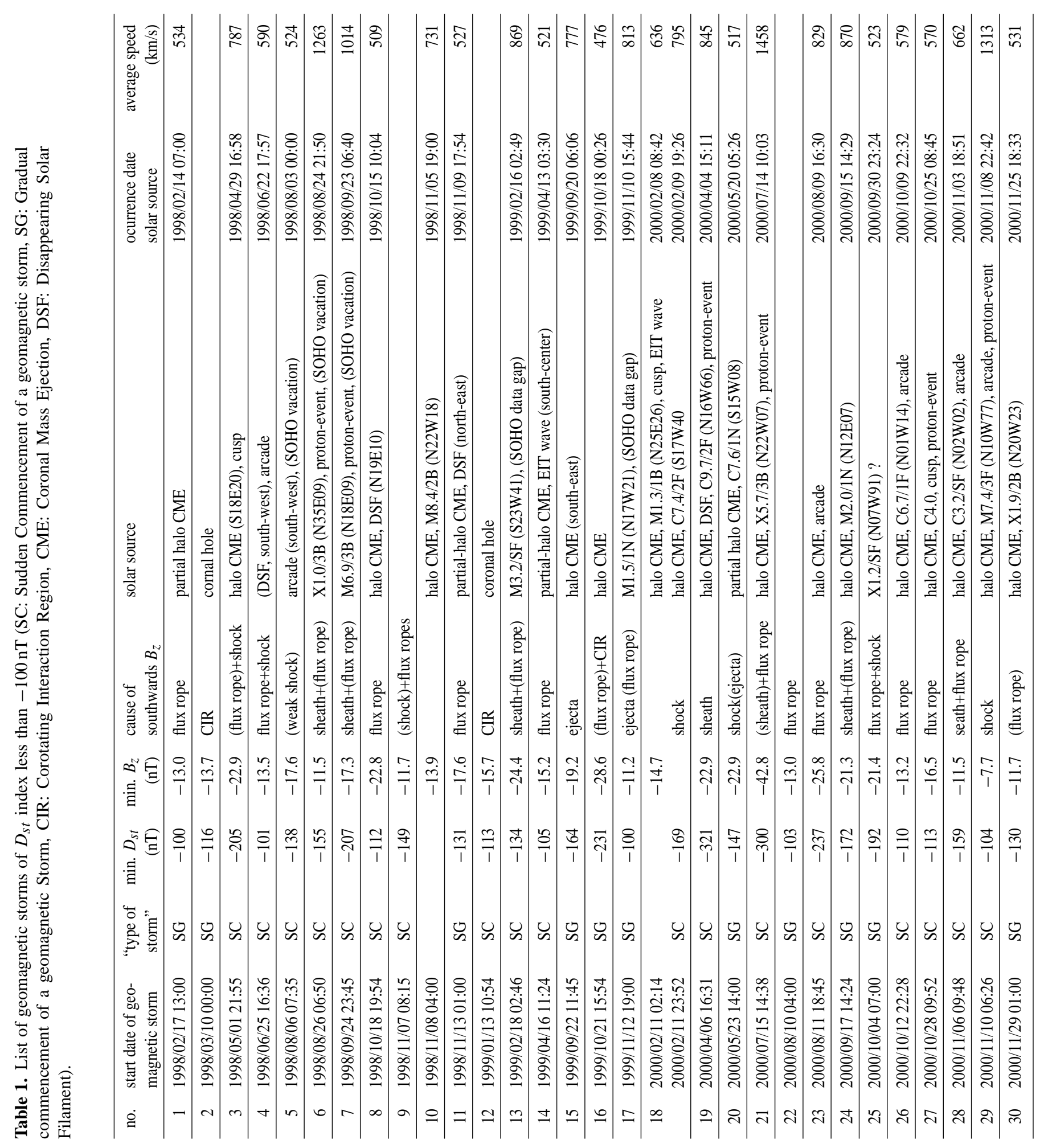


We first investigated the relationship between the transit speeds of the interplanetary disturbances and the minimum values of the $D_{s t}$ indices during the geomagnetic storms. Figure 1 shows a scatter plot of these parameters for all of the storms listed in Table 1 for which solar sources were identified, sorted on the interplanetary drivers. Squares, triangles, and diamonds represent the storms associated mainly with interplanetary magnetic flux ropes, sheath regions (or shocks), and interactions, respectively. According to Fig. 1, a correlation between the speed and the magnitude of geomagnetic storms is not clear. The correlation is weak even though the magnetic storms were associated with sheath regions or interactions. This result is consistent with the result by Cane, Richardson, and St. Cyr (2000) (see their Fig. 4).

\section{Cause of strong southward IMF}

In this section we will discuss the various interplanetary causes of the southward $B_{z}$ listed in Table 1, illustrating the categories with examples of the interplanetary data together, with the $D_{s t}$ signatures.

\subsection{Corotating Interaction Regions (CIRs)}

Geomagnetic storms of numbers 2 and 12 listed in Table 1 were associated with CIRs. The number of geomagnetic storms associated with CIRs was small because the studied period was near the maximum of solar cycle 23. Many geomagnetic storms caused by CIRs are usually observed during the declining phase of solar cycles (Watari, 1997; Watari and Watanabe, 1998).

\subsection{Southward IMFs associated with magnetic flux ropes}

According to Table 1, the magnetic flux ropes were observed in 21 of 30 geomagnetic storms. And they were the main cause of strong southward IMF in 10 out of 30 geomagnetic storms.

Figure 2 shows $D_{s t}$ index and one-hour-shifted solar wind parameters (magnetic fields, speed, density, temperature, $\beta$, and ratio of helium and hydrogen) of storm no. 21 on 15 July 2000. The solar wind parameters were measured at the L1 point, approximately $1.5 \times 10^{6} \mathrm{~km}$ upstream from the Earth. Hence, we plotted the one-hour-shifted values to compare them with the $D_{s t}$ index observed at the Earth. Two vertical solid lines in Fig. 2 indicate the start and end times of the geomagnetic storm. The dashed curve plotted in the temperature panel shows the temperature calculated from the solar wind speed by using the correlation between solar wind speed and proton temperature (Lopez, 1987). The abnormally depressed proton temperature compared with the calculated temperature has often been observed in the case of interplanetary magnetic flux ropes or ejecta (Richardson and Cane, 1993). Low $\beta$ or higher ratios of helium and hydrogen were other signatures of ejecta (Goldstein, Neugebauer, and Clay, 1998).

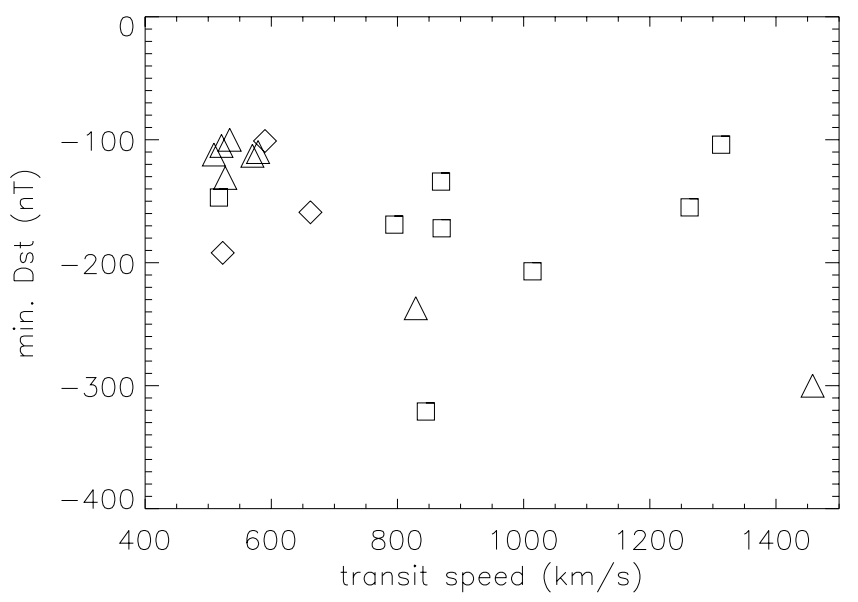

Fig. 1. A scatter plot of average transit speed of interplanetary disturbances and minimum $D_{s t}$ index of geomagnetic storms (square: magnetic flux rope, triangle: shock, and diamond: interaction).

Storm no. 21 is an example of a storm caused by a magnetic flux rope with a strong axial magnetic field (approximately $80 \mathrm{nT}$ ) and high speed (more than $1000 \mathrm{~km} / \mathrm{s}$ ). This magnetic flux rope was associated with the halo CME on 14 July 2000 (the so-called "Bastille Day event") and produced an intense geomagnetic storm. Figure 3 shows a negative image of the soft X-ray solar observation associated with this event. This image was taken by the Yohkoh/Soft X-ray Telescope (SXT). The soft X-ray arcade expanded in the eastwest direction. According to the result of a fitting of the cylindrical magnetic flux model (Marubashi, 1997) to the solar wind data, the orientation of the magnetic flux rope was longitude, 34 degrees and latitude, 10 degrees. The projected direction of the magnetic flux rope on the solar surface (the arrow in Fig. 3) is close to the orientation of the observed soft $\mathrm{X}$-ray arcade. This suggests that the orientation of the magnetic flux rope stayed constant during propagation, and there is a possibility to infer southward IMF inside the magnetic flux rope based on the orientation of soft X-ray arcades or on the solar observations. Watari, Watanabe, and Marubashi (2001) noted that orientations of interplanetary magnetic flux ropes correspond well to the orientation of a stretch soft Xray solar activities or soft $\mathrm{X}$-ray arcades).

\subsection{Interaction between a magnetic flux rope and a CIR}

Figure 4 shows the solar wind parameters and $D_{s t}$ index of storm no. 16 on 21 October 1999. According to this figure, southward IMF in the slow-speed ejecta was strengthened by the following high-speed solar wind or a CIR. This kind of event has been reported by Watari and Watanabe (1998).

3.4 Interaction between a magnetic flux rope and a following shock

In storm no. 3 a weak geomagnetic storm associated with a magnetic flux rope already started (Fig. 5). This mag- 


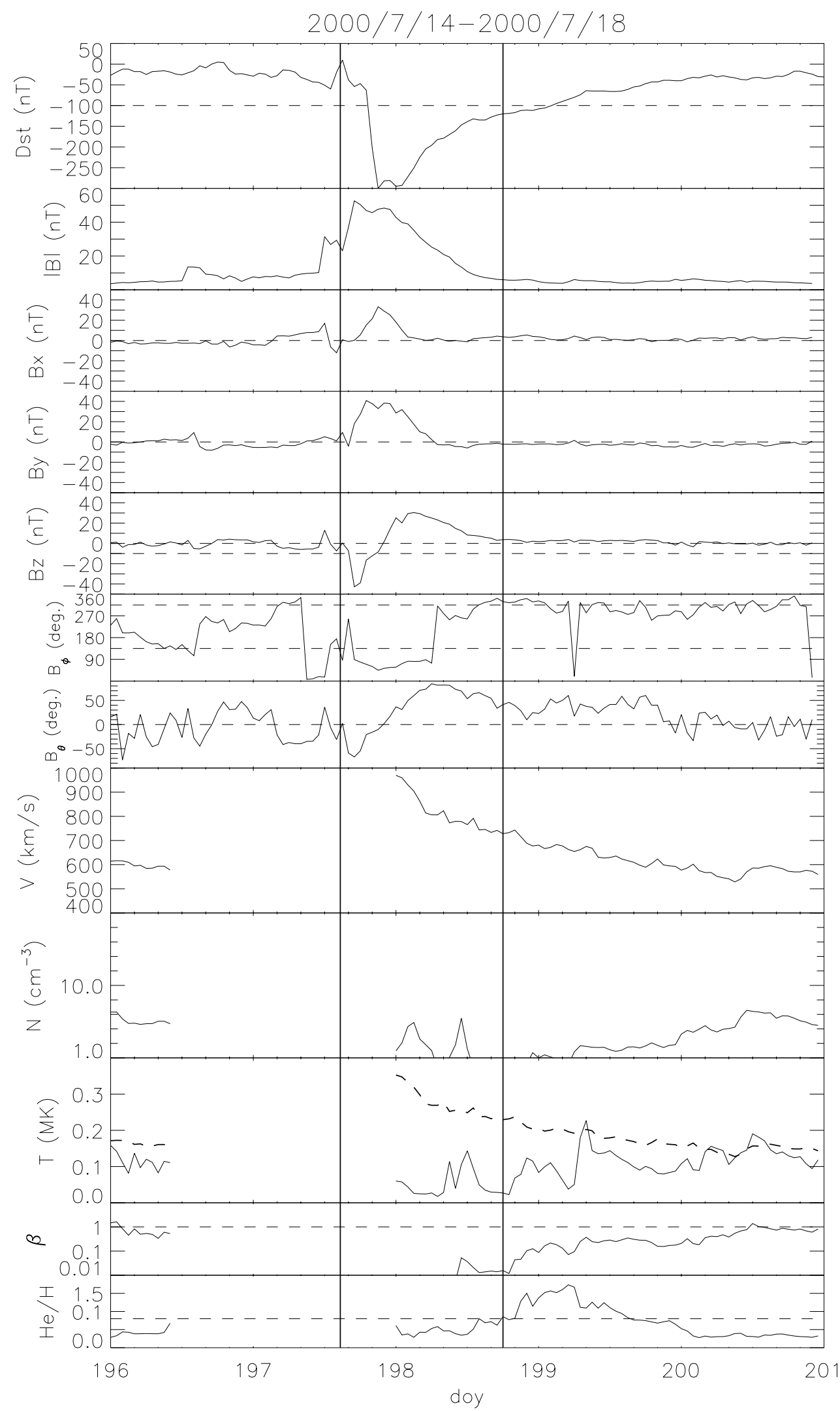

Fig. 2. $D_{s t}$ index and solar wind parameters of storm no. 21. Two vertical solid lines show the duration of the geomagnetic storm. Horizontal dashed lines stress some distinct values ( $D_{s t}$ index: $-100 \mathrm{nT}, B_{z}:-10 \mathrm{nT}$, B-phi: 135 and 315 degrees, $\beta: 1$, and $\mathrm{He} / \mathrm{H}: 0.08$ ). 


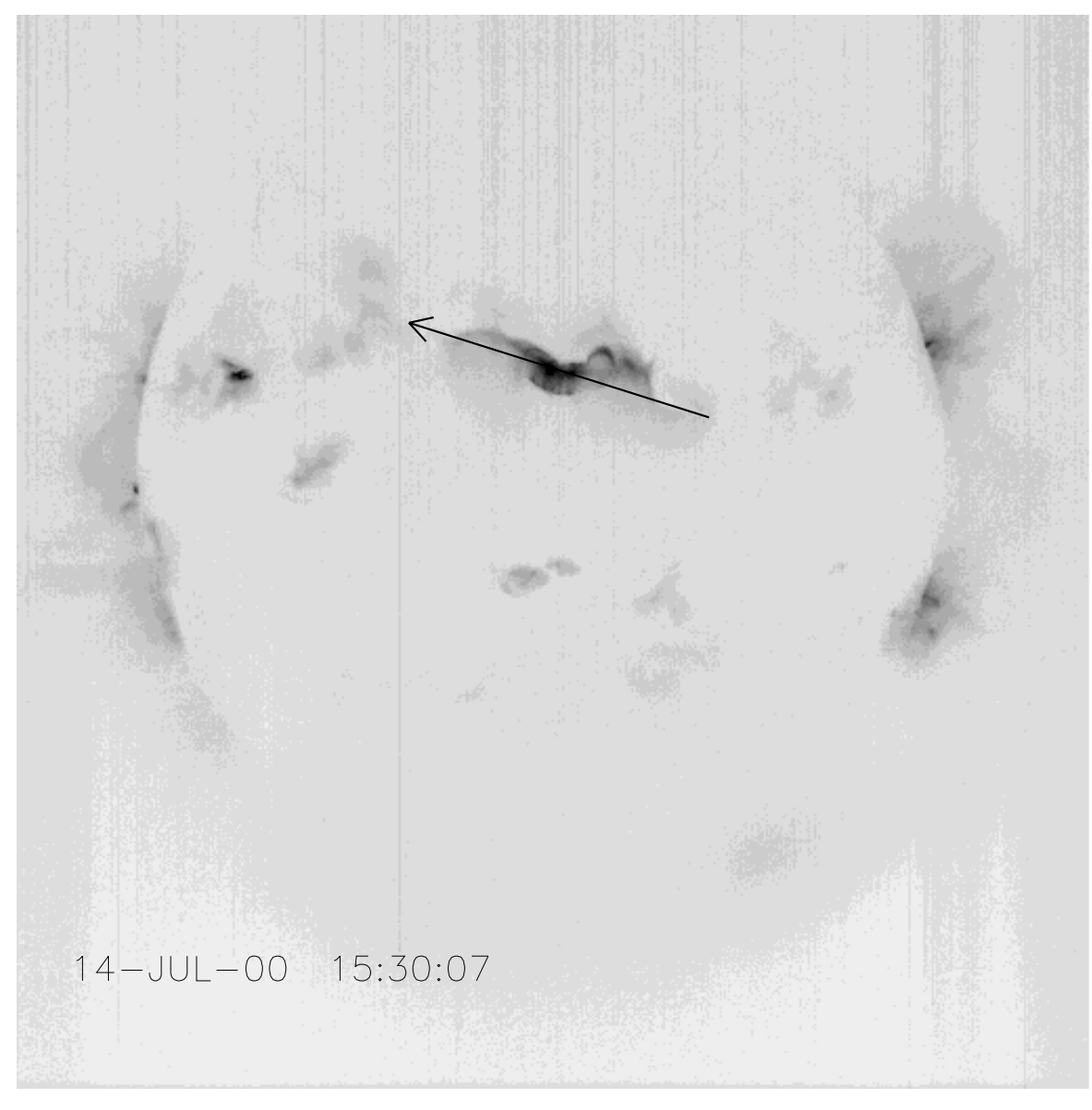

Fig. 3. A negative image of soft $\mathrm{X}$ ray observation of solar activity associated with storm no. 21. This image was taken by the Yohoh/SXT. The arrow shows the projected orientation of the magnetic flux rope observed by the ACE spacecraft. netic flux rope was associated with a halo CME on 29 April 1998. And then another strong shock arrived at the Earth. The strong southward IMF associated with this shock made the storm more intense. This is an example of a double-step storm (Gonzales et al., 2001).

The strong southward IMF of storm no. 4 was observed behind a magnetic flux rope (Fig. 6). In this case, the flux rope itself did not carry the strong southward component of IMF. A shock that penetrated into the flux rope possibly produced the strong southward IMF.

In storm no. 9, there were two major dips in the $D_{s t}$ index, as shown in Fig. 7. The first dip corresponded to the southward IMF of an ejection in front of a strong shock, and the second dip corresponded to the southward IMF caused by a magnetic flux rope.

Figure 8 shows the plots for storm no. 20 on 23 May 2000. In this case, the southward IMF was formed between two ejecta. Figure 8 indicates that the collision of the ejecta strengthened the southward IMF.

\subsection{Southward IMF in sheath}

In storm no. 13, strong southward IMF of more than $20 \mathrm{nT}$ was observed on 18 February 1999. This strong southward IMF was formed in the sheath region, as shown in Fig. 9.
This event was associated with the M3.2/SF flare that occurred at S23W41. Figure 10 shows a source surface map produced by the Wilcox Solar Observatory. The solar source of the storm (a circle in Fig. 10) was located in the southern solar hemisphere in the away sector and an upper part of ejecta was expected to pass the Earth. The southward IMF in front of ejecta in the upper panel of Fig. 13 can explain the southward IMF of this event. This example suggests that it is possible to infer the southward IMF expected at $1 \mathrm{AU}$ according to the solar observations.

Figure 11 shows the plot for storm no. 24 on 17 September 2000. Two dashed lines show a duration of a magnetic flux rope. A high-speed interplanetary disturbance with a speed of more than $800 \mathrm{~km} / \mathrm{s}$ formed a sheath region with strong southward IMF, and the geomagnetic storm began at the same time as the arrival of this disturbance. Then there was a magnetic flux rope behind the sheath region. However, this magnetic flux rope did not have strong southward IMF in this case.

\subsection{Miscellaneous}

Figure 12 shows the $D_{s t}$ indices and the solar wind parameters of storm no. 5. The southward IMF continued for approximately twelve hours. Associated with a weak shock, 


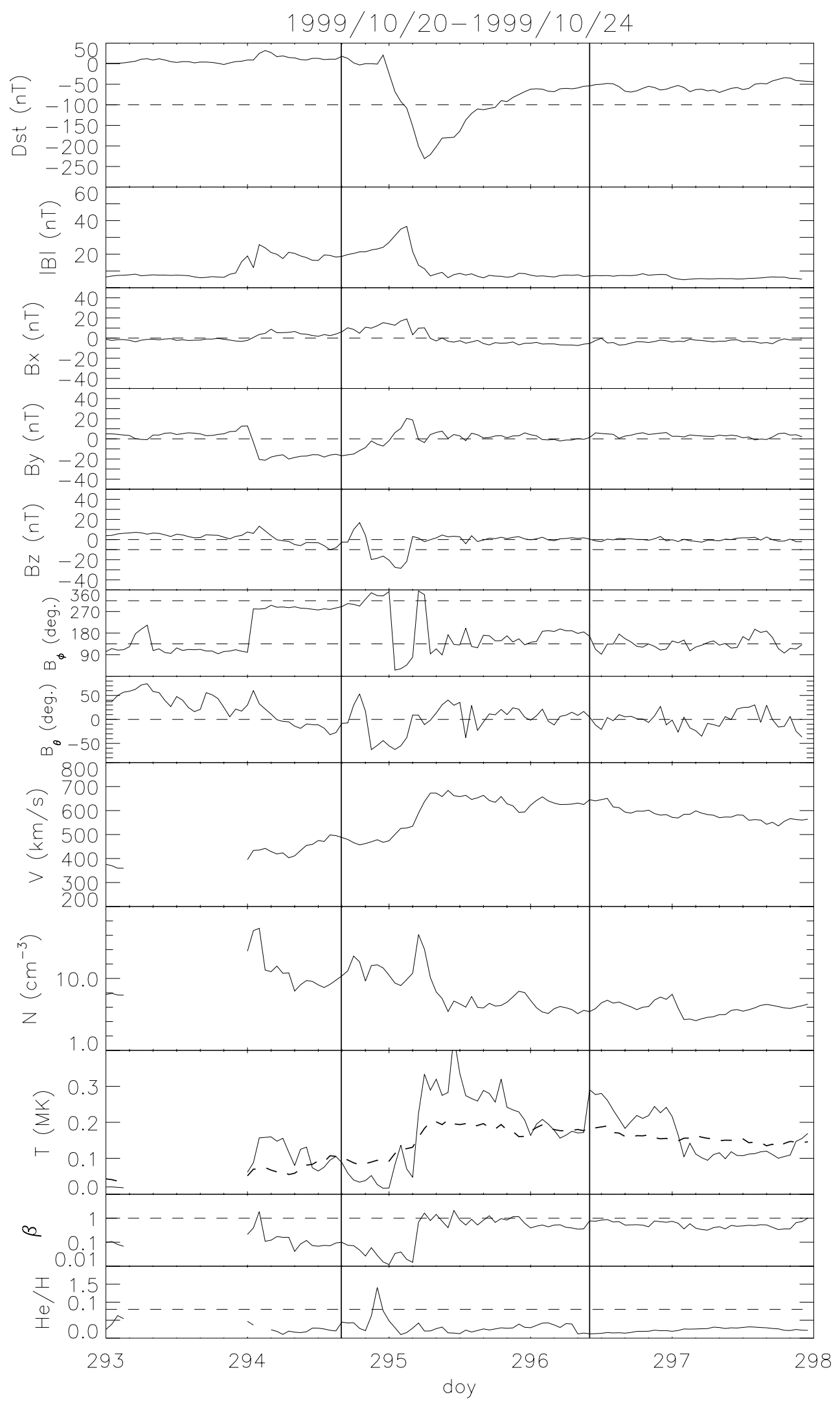

Fig. 4. $D_{s t}$ index and solar wind parameters of storm no. 16. Two vertical solid lines show the duration of the geomagnetic storm. 


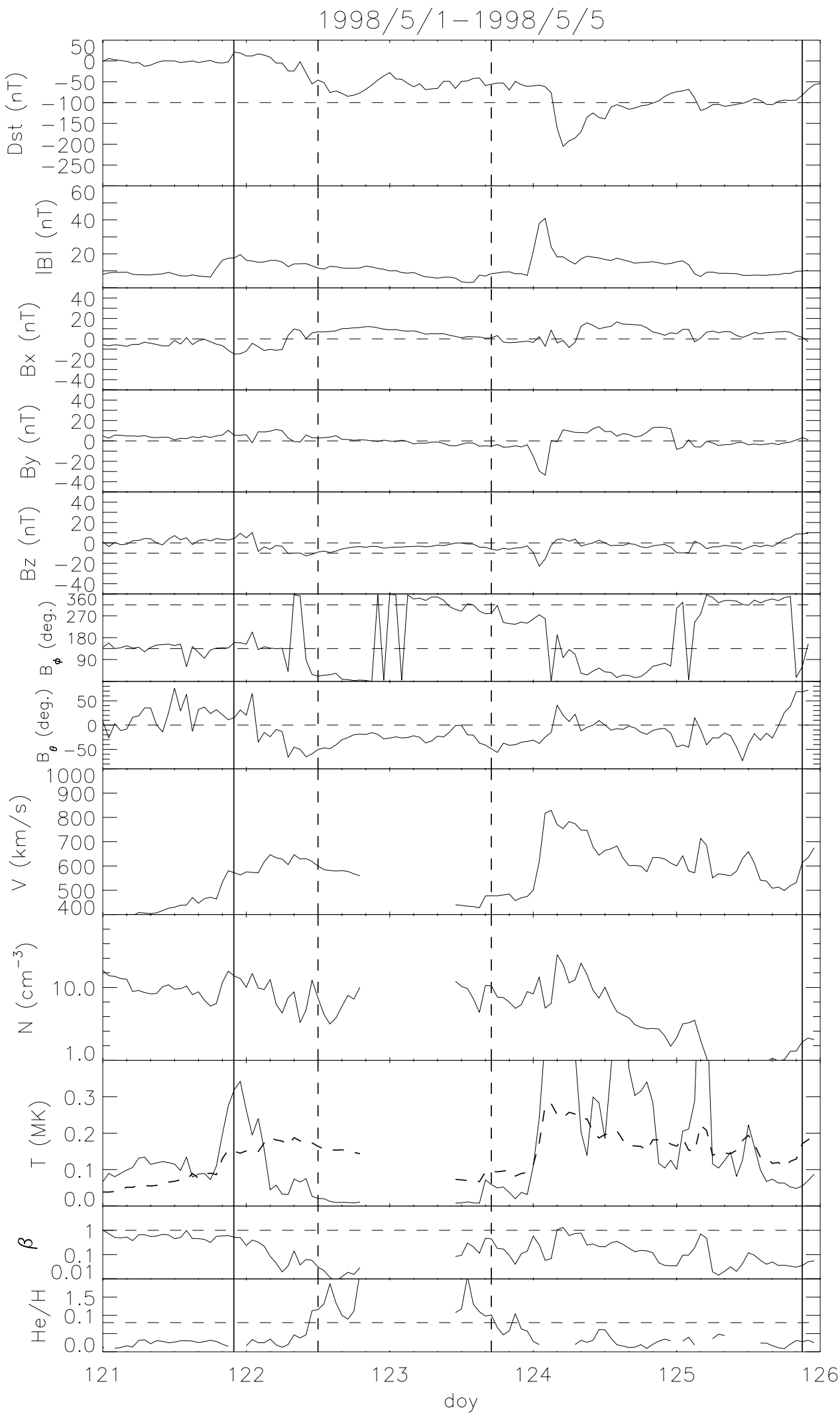

Fig. 5. $D_{s t}$ index and solar wind parameters of storm no. 3. Two vertical solid lines show the duration of the geomagnetic storm and two vertical dash lines show the duration of a magnetic flux rope. 


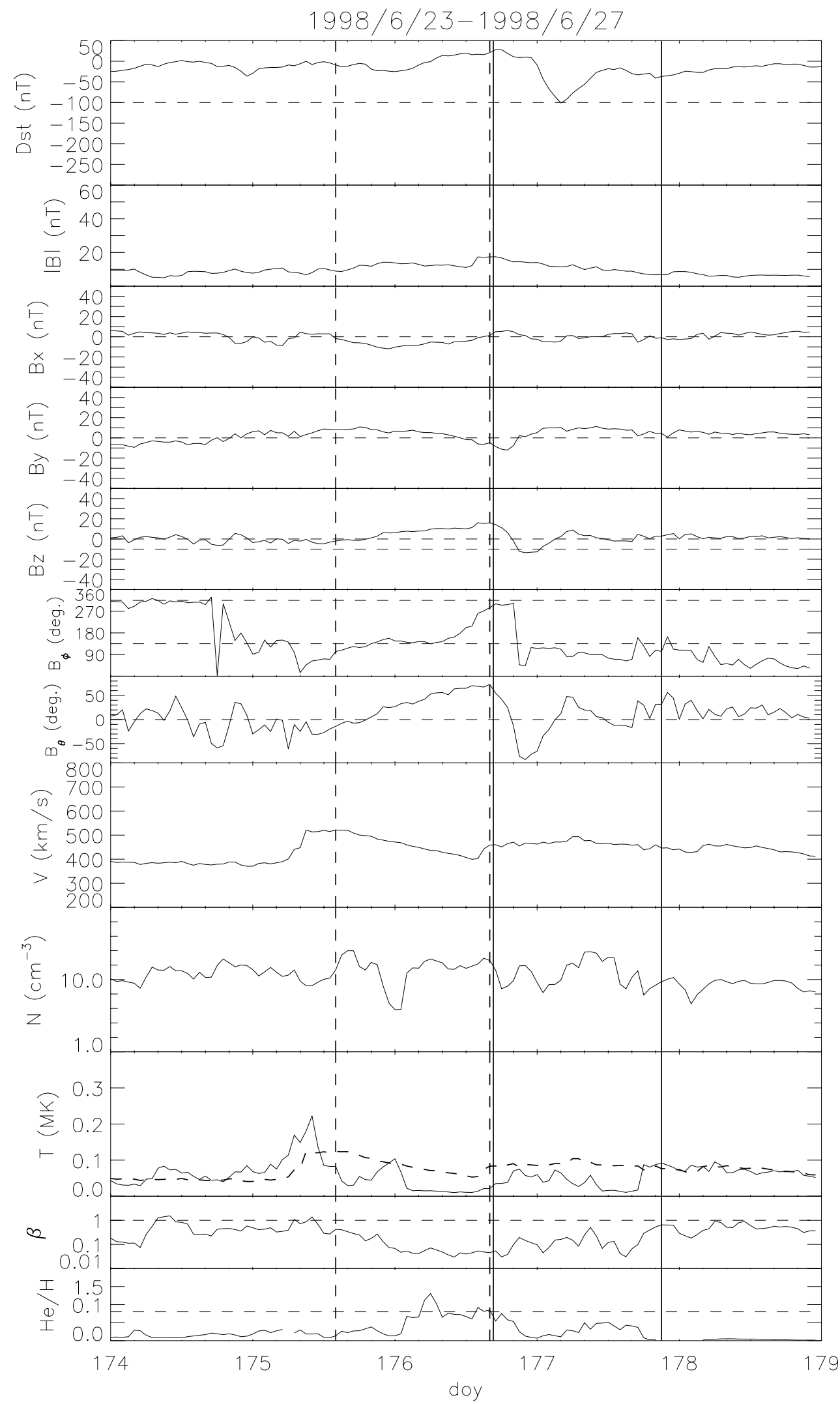

Fig. 6. $D_{s t}$ index and solar wind parameters of storm no. 4. Two vertical solid lines show the duration of the geomagnetic storm and two vertical dash lines show the duration of a magnetic flux rope. 


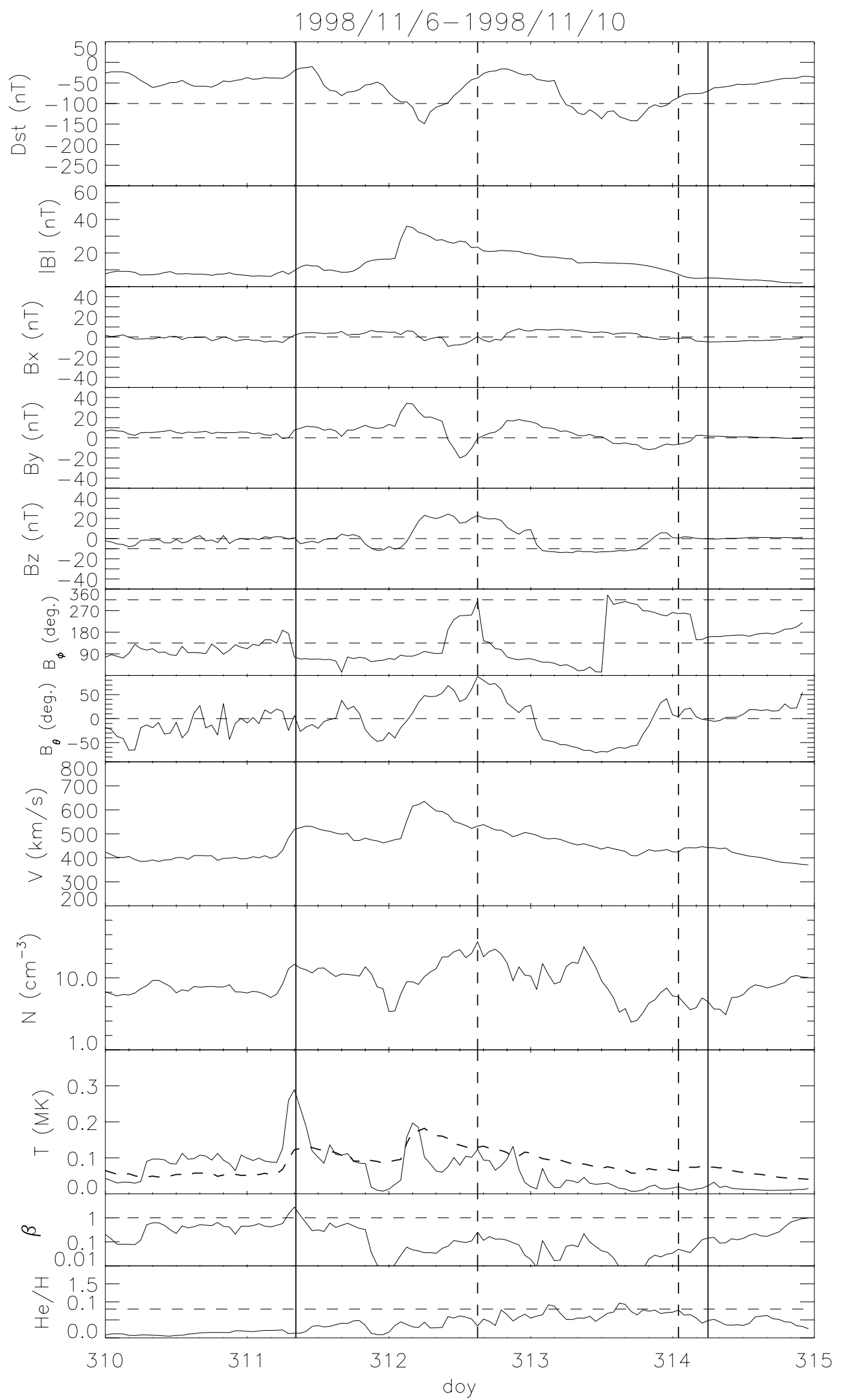

Fig. 7. $D_{s t}$ index and solar wind parameters of storm no. 9. Two vertical solid lines show the duration of the geomagnetic storm and two vertical dash lines show the duration of a magnetic flux rope. 


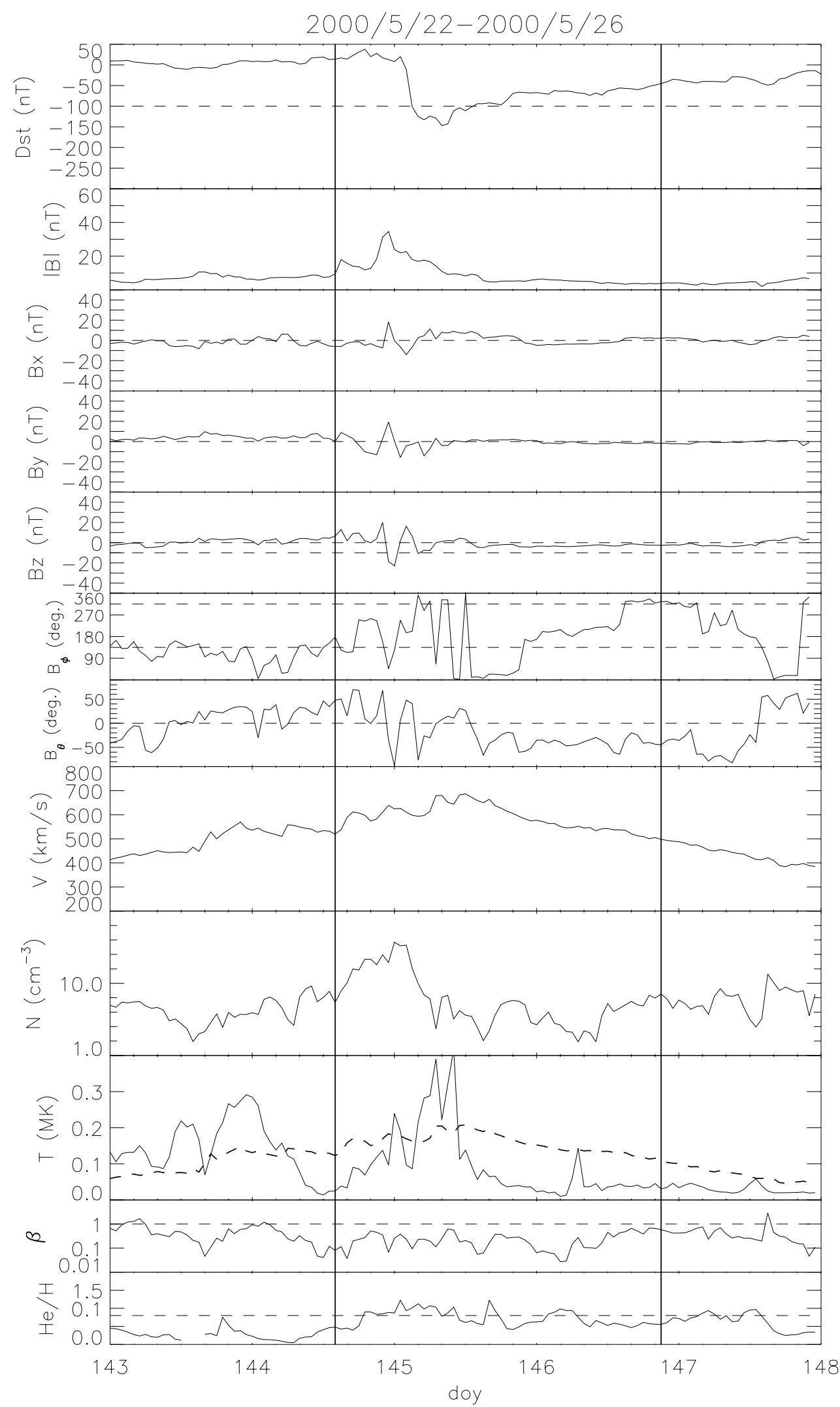

Fig. 8. $D_{s t}$ index and solar wind parameters of storm no. 20. Two vertical solid lines show the duration of the geomagnetic storm. 


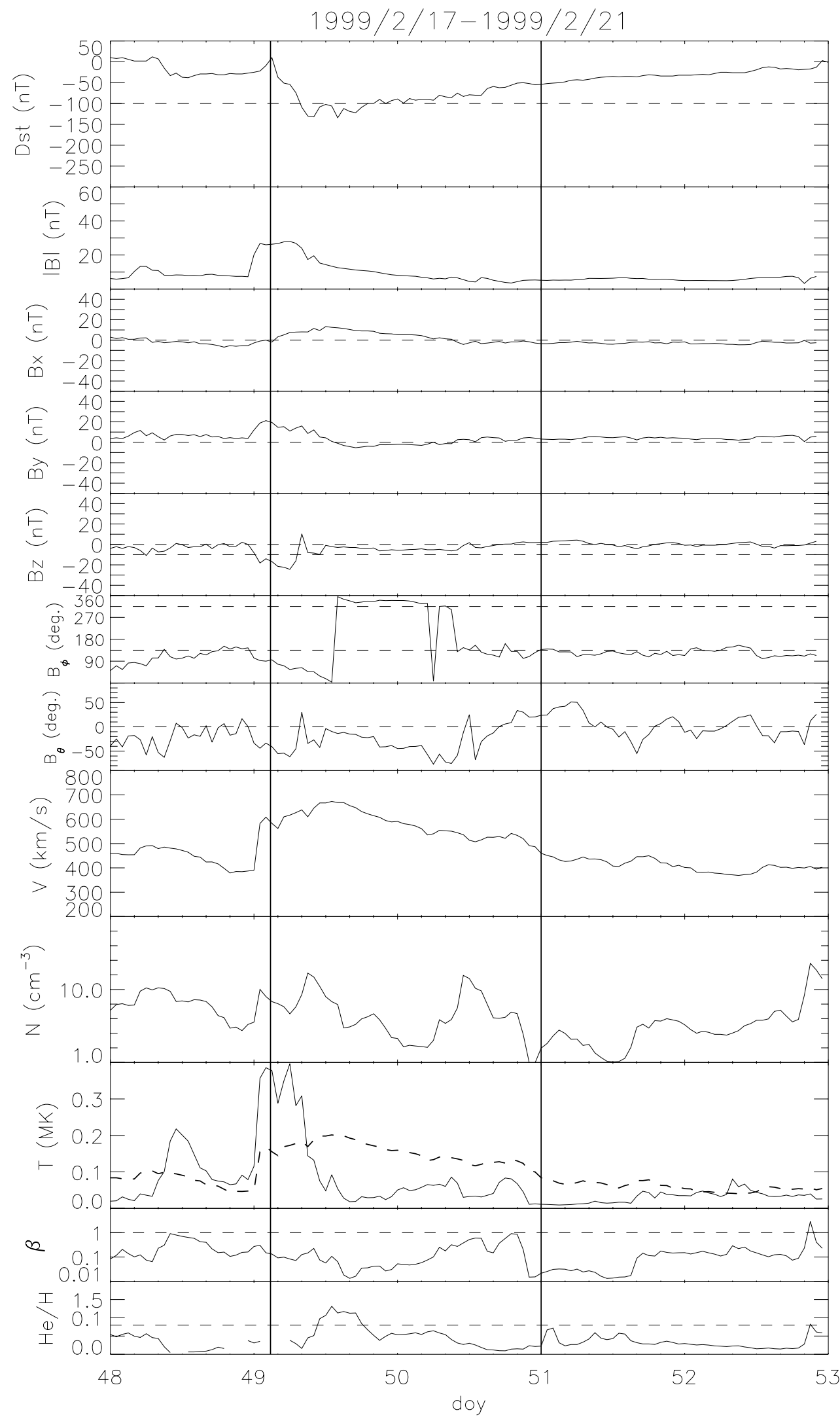

Fig. 9. $D_{s t}$ index and solar wind parameters of storm no. 13. Two vertical solid lines show the duration of the geomagnetic storm. 


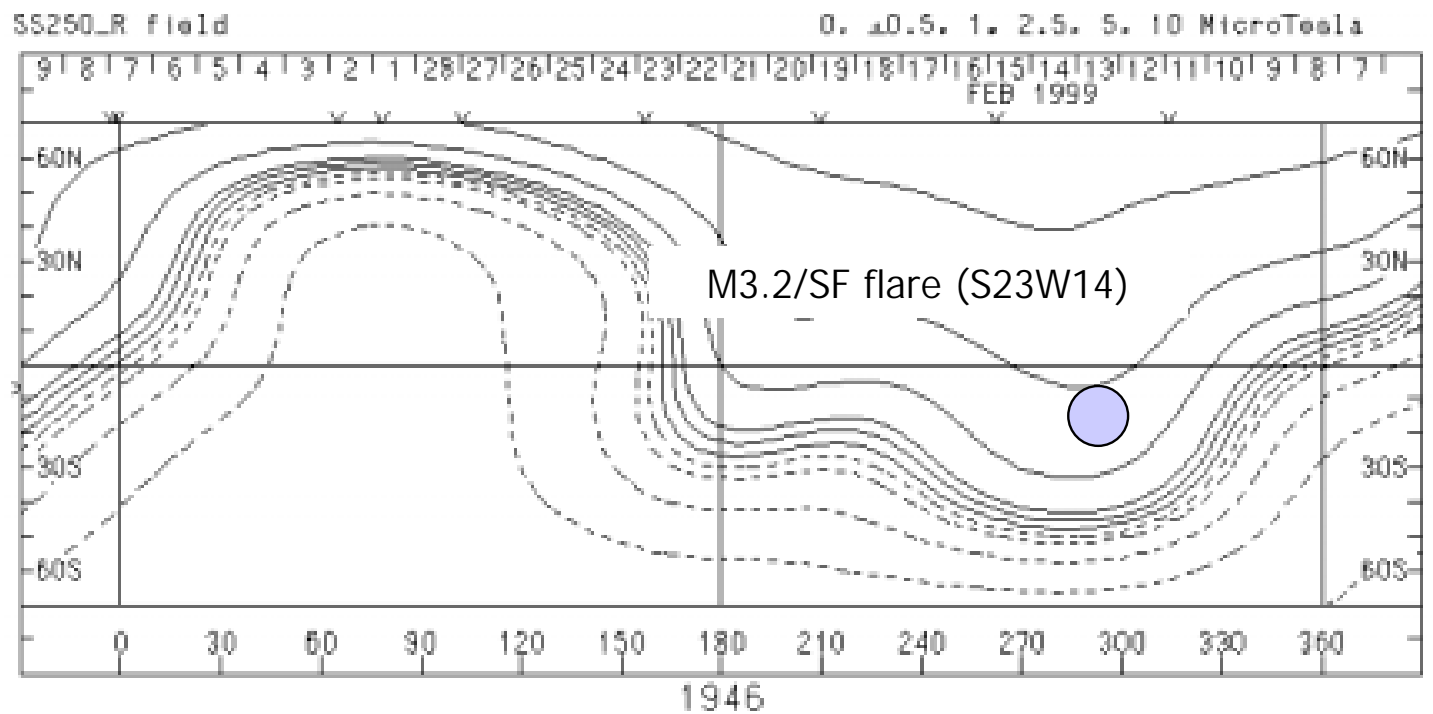

Fig. 10. A source surface map produced by the Wilcox Solar Observatory. The circle shows the location of a M3.2/SF flare associated with the geomagnetic storm no. 13 .

the magnitude of IMF increased to approximately $20 \mathrm{nT}$. The speed of the solar wind was approximately $400 \mathrm{~km} / \mathrm{s}$ during this period. In storms numbers 15 and 17 , strong southward IMFs were observed in low temperature ejecta. However, causes of these southward IMFs were uncertain.

\section{Possibility to infer southward IMF}

There are two main causes of the formation of southward IMF associated with transient events. One possible source is a southward component directly ejected from the Sun as interplanetary magnetic flux ropes. Another is a formation of southward IMF by an interaction between an interplanetary disturbance and the background solar wind or between interplanetary disturbances.

It is possible, in some cases, to infer orientation of a magnetic flux rope from solar observations (orientation of solar filaments that disappeared or soft X-ray arcades), by assuming that the orientation of the flux rope remains constant during propagation (see Sect. 3.2, storm no. 21). This means that it is possible to infer southward IMF inside the flux rope.

There are four ways to form southward IMF by interaction between ejecta and the IMF of the background solar wind (Fig. 13). In this figure, the ejecta travel from left to right. The southward IMF that forms in front of ejecta is well known (McComas et al; 1989; Wu, Dryer, and Wu, 1996). In contrast, we found the formation of southward IMF in the tail of the ejecta through the interaction with the following CIRs or shocks (see Sects. 3.3 and 3.4). According to Fig. 13, it is possible to infer southward IMF by using the sector of background solar wind if we know which part (e.g. upper part or lower part) of the ejecta passes the Earth. The upper part of the ejecta tends to pass the Earth if the ejecta originates in the southern hemisphere of the Sun. On the other hand, the lower part of the ejects tends to pass the Earth if the ejecta originates in the northern hemisphere of the Sun. For example, southward IMF in front of the ejecta observed at $1 \mathrm{AU}$ is formed in the away sector in the upper part of the ejecta and in the towards sector in the lower part of the ejecta (see Sect. 3.5).

\section{Discussion and summary}

Southward IMF is an important factor in the intensity and duration of geomagnetic disturbances. One purpose of this study was to find a way to infer south $B_{z}$ two- or three-days in advance. It was found that the strong southward IMF is either ejected from the Sun as interplanetary magnetic flux ropes, or it is formed by the interaction between an interplanetary disturbance and background solar wind or interplanetary disturbances. We presented a possibility of inferring southward IMF from solar observations considering those mechanisms.

According to our study, intense geomagnetic storms often occurred in association with interplanetary magnetic flux ropes. However, strong southward IMFs were observed around the interplanetary flux ropes rather than inside them. They were formed through the interaction of the flux ropes with the background solar wind. Interactions between interplanetary disturbances, or between the other interplanetary disturbances and the background solar wind also produced strong southward IMFs. This is an important factor in the severity of storms. The interaction causes the observed complex signatures of interplanetary disturbances (see Sect. 3). And the interactions make it more difficult to infer southward IMF at $1 \mathrm{AU}$ from solar observations. 


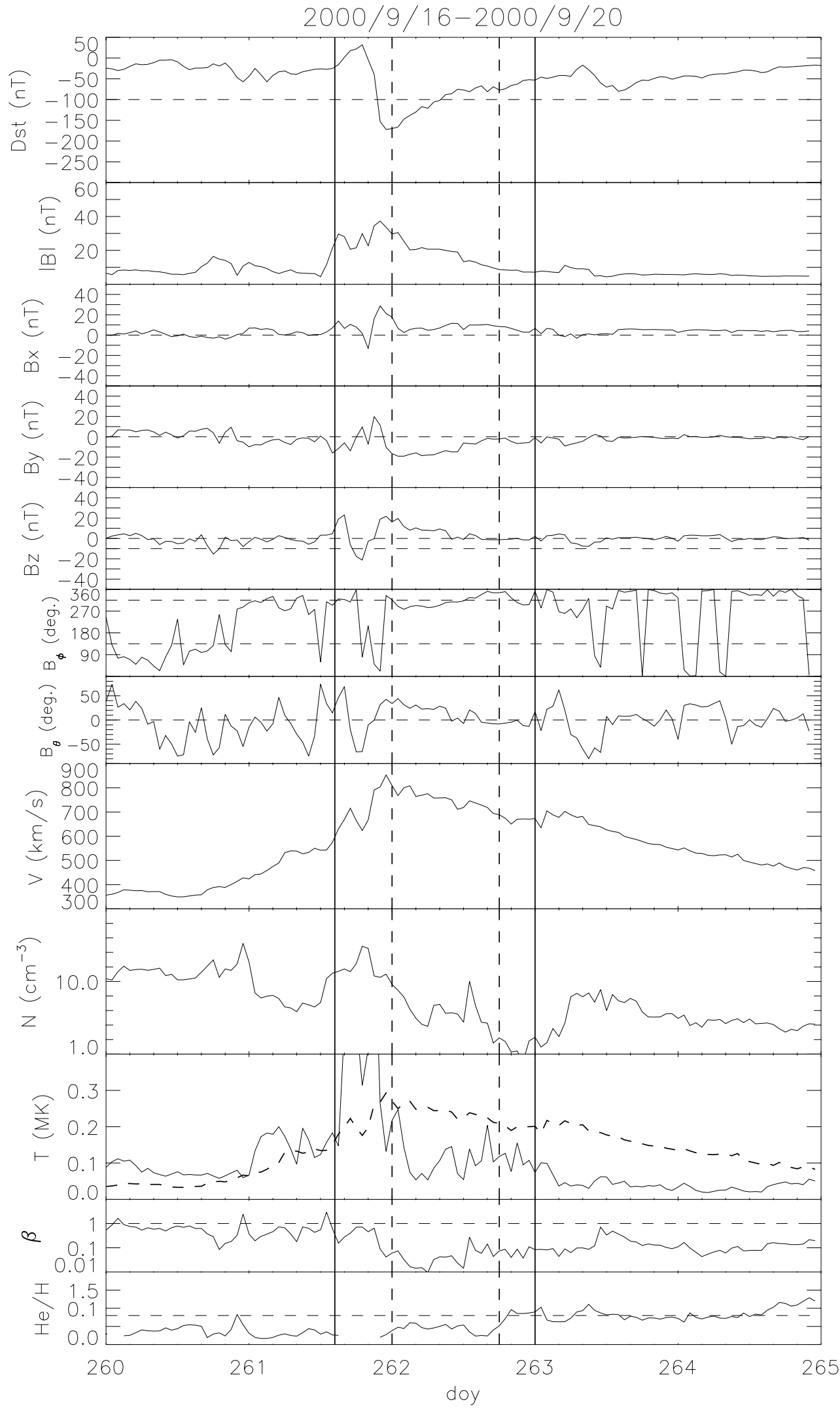

Fig. 11. $D_{s t}$ index and solar wind parameters of storm no. 24. Two vertical solid lines show the duration of the geomagnetic storm and two vertical dash lines show the duration of a magnetic flux rope. 


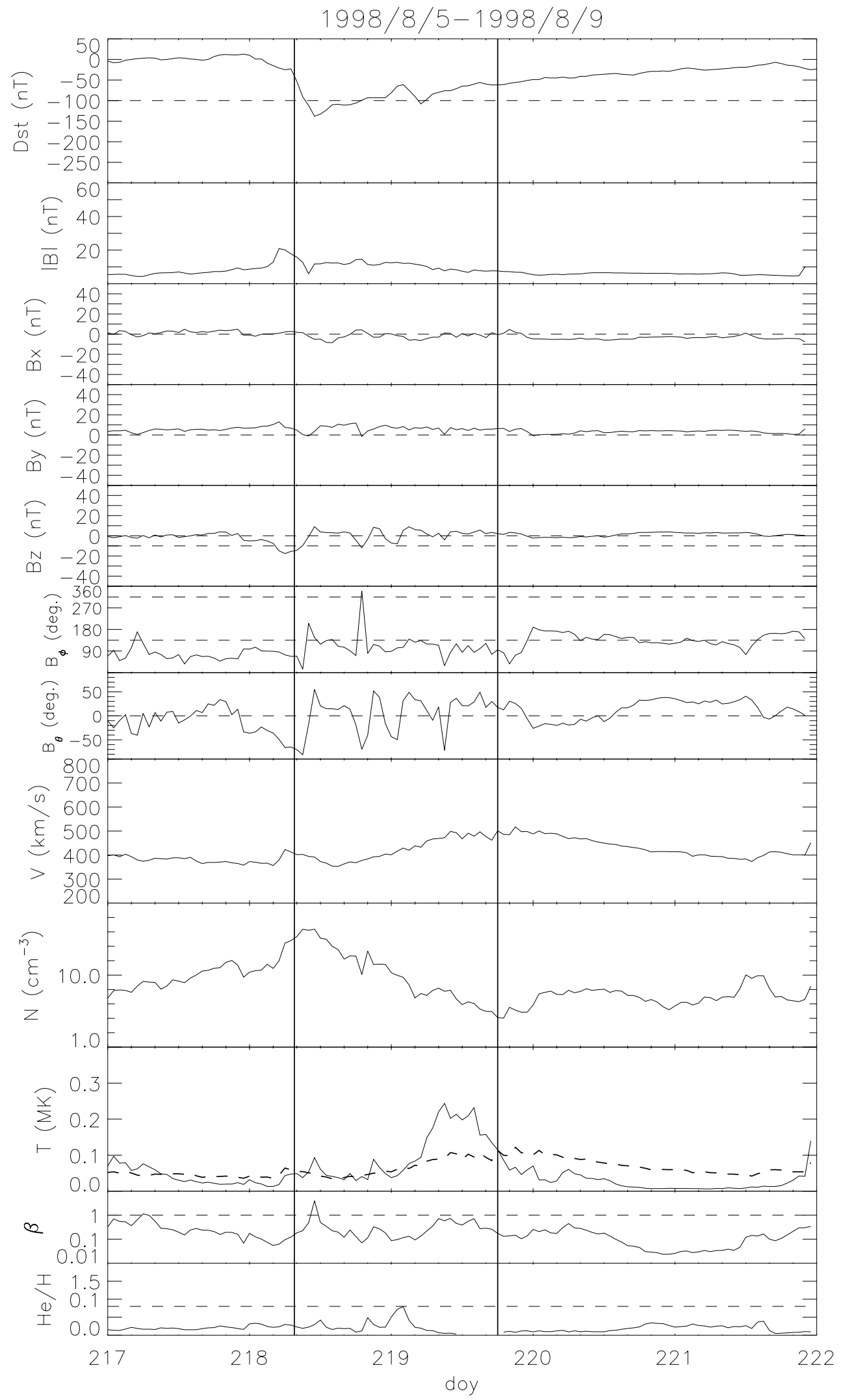

Fig. 12. $D_{s t}$ index and solar wind parameters of storm no. 5. Two vertical solid lines show the duration of the geomagnetic storm. 


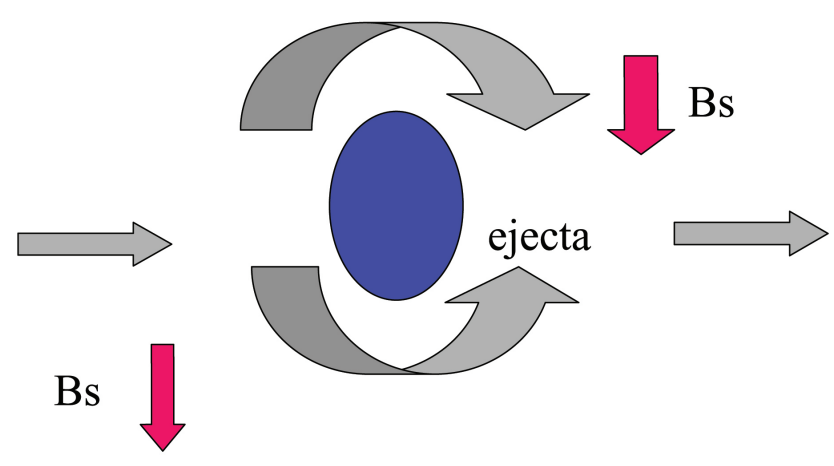

(a) away sector

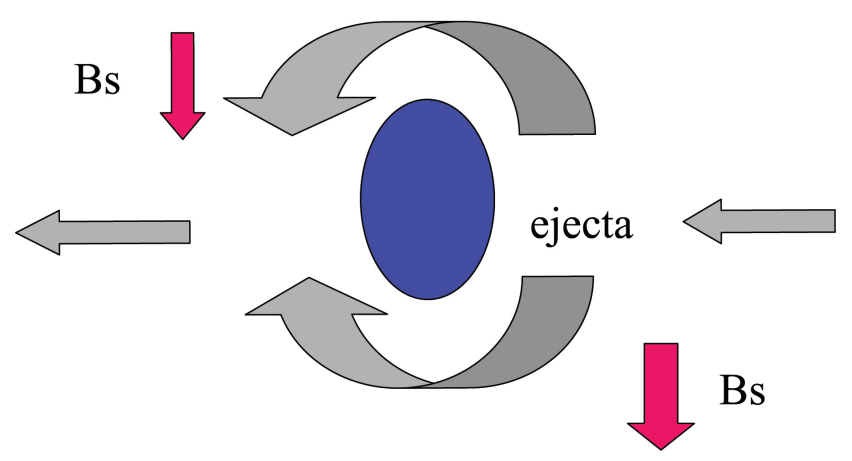

(b) toward sector

Fig. 13. Formation of southward IMF through the interaction between ejecta and IMF of the background solar wind. The upper panel shows the away sector and the lower panel shows the toward sector. The Sun is on the left-hand side, right is the direction towards the Earth, the horizontal gray arrow indicates the direction of the interplanetary magnetic field, the broad gray arrows indicate the direction of the field draped around the ejecta. The ejecta travel from the left side to the right side in this figure. " $B s$ " means southward IMF.

Acknowledgements. We would like to thank the ACE Science Center for the use of solar wind data measured by the ACE spacecraft, D. J. McComas (Los Alamos National Laboratory) for the ACE/SWEPAM data, and N. F. Ness, L'Heureux, and C. W. Smith (Bartol Research Institute) and L. Burlaga and M. Acuña (NASA/GSFC) for the ACE/MAG data. SOHO is a joint project of NASA and ESA. Soft X-ray solar images were provided from the Yohoh/SXT team. One of us (M.V.) was supported by project S1003006 form the Academy of Sciences of the Czech Republic.

The Editor in Chief thanks Z. Smith for his help in evaluating this paper.

\section{References}

Cane, H., Richardson, I. G., and St. Cyr, O. C.: Coronal mass ejections, interplanetary ejecta and geomagnetic storms, Geophys. Res. Lett., 27, 5391, 2000.

Goldstein, R., Neugebauer, M., and Clay, D.: A statistical study of coronal mass ejection plasma flows, J. Geophys. Res., 103, 4761, 1998.

Gonzalez, W. D., de Gonzaleze, C., Sobral, J. H. A., Lago, A. D., and Vieira, L. E.: Solar and interplanetary causes of very intense geomagnetic storms, J. Atmos. Solar-Terr. Phys., 63, 403, 2001.

Lopez, R.: Solar cycle invariance in solar wind proton temperature relationships, J. Geophys. Res., 92, 11 189, 1987.

Marubashi, K.: Interplanetary magnetic flux ropes and solar filaments, in: Coronal Mass Ejections, Geophys. Monogr. Ser., Vol. 99, edited by Crooker, N., Joselyn, J. A., and Feynman, J., p. 147, AGU, Washington, D. C., 1997.

McComas, D. J., Gosling, J. T., Bame, S. J., Smith, E. J., and Cane, H. V.: A test of magnetic field draping induced $B_{z}$ perturbations ahead of fast coronal mass ejecta, J. Geophys. Res., 94, 1465, 1989.

Richardson, I. G. and Cane, H. V.: Signatures of shock drivers and their dependence on the solar source location, J. Geophys. Res., 98, $15295,1993$.

Tsurutani, B. J., Gonzalez, W. D., Tang, F., Akasofu, S. I., and Smith, E. J.: Origin of interplanetary southward magnetic fields responsible for major magnetic storms near solar maximum (1978-1979), J. Geophys. Res., 93, 8519, 1988.

Tsurutani, B. and W. Gonzalez: The interplanetary causes of magnetic storms: A review, in: Magnetic Storms, edited by Tsurutani, B. T., Gonzalez, W. D., Kamide, Y., and Arballo, J. K., Geophys. Mon. Series, 98, 77-89, Washington D. C., 1997.

Watari, S.: The effect of the high speed stream following the corotating interaction region: Analysis of large south polar coronal holes observed between December 1993 and June 1994 and longduration geomagnetic disturbances, Ann. Geophysicae, 15(6), 662-670, 1997.

Watari, S. and Watanabe, T.: The solar drivers of geomagnetic disturbances during solar minimum, Geophys. Res. Lett., 25, 2489, 1998.

Watari, S., Watanabe, T., and Marubashi, K.: Soft X-ray activities associated with interplanetary magnetic flux ropes, Solar Phys., 202, 363, 2001.

Wu, C.-C., Dryer, M., and Wu, S. T.: Three-dimensional MHD simulation of interplanetary magnetic field changes at $1 \mathrm{AU}$ as a consequence of simulated solar flares, Ann. Geophysicae, 14, 383, 1996.

Wu, C.-C., Dryer, M., Smith, Z., Wu, S. T., and Lyu, L. H.: Recipe for predicting the IMF $B_{z}$ polarity's change of direction following solar disturbances and the onset of geomagnetic storms, J. Atmos. Solar-Terr. Phys., 58, 1805, 1996. 